\title{
Poisson distribution for a sum of additive functions on shifted primes
}

by

Jonas Šiaulys and Gediminas Stepanauskas (Vilnius)

1. Introduction. The function $f$ defined on the positive integers is said to be additive if

$$
f(m n)=f(m)+f(n) \quad \text { for }(m, n)=1, \quad f(1)=0 .
$$

If, in addition,

$$
f\left(p^{k}\right)=f(p)
$$

for all prime powers $p^{k}$, then $f$ is called strongly additive. In our paper the additive functions always depend on a real parameter $x$, therefore we will write $f_{x}$.

The weak convergence, as $x \rightarrow \infty$, of the distributions

$$
\nu_{x}\left(n \leq x, f_{x}(A(n))<u\right):=\frac{1}{[x]} \sum_{\substack{n \leq x \\ f_{x}(A(n))<u}} 1,
$$

where $A(n)=n$, is quite well explored (see monographs [2], [3], [7]). Other cases, when $A(n)$ is an arithmetically interesting sequence of positive integers, are more difficult and less considered. But there are rather general results when $A(n)$ runs through polynomial values or through a set of shifted primes (for related references see [2], [6]). For the study of the limit distribution of (1), various methods were used. Some results proved by sieve methods, by approximating an additive function by a sum of independent random variables, or by the factorial moments method can be generalized to sums of two or more additive functions with shifted arguments (see [8] for references). And this can be done without changing the method of proof provided the arithmetic structure of $A(n)$ is not too difficult. In [8], [9] we

2000 Mathematics Subject Classification: Primary 11N37, 11N60.

Key words and phrases: additive arithmetic function, Poisson distribution, factorial moments. 
considered the weak convergence of the distributions

$$
\nu_{x}\left(p \leq x, f_{x}(p+1)<u\right)
$$

and

$$
\nu_{x}\left(n \leq x, f_{x}(n)+g_{x}(n+1)<u\right)
$$

to the Poisson law.

In the present article we continue the investigations of [8], [9] and obtain necessary and sufficient conditions for the weak convergence, as $x \rightarrow \infty$, of the distributions

$$
\nu_{x}\left(p \leq x, f_{x}(p+1)+g_{x}(p+2)<u\right)
$$

to the Poisson law. The Poisson law as the limit distribution for the sum of additive functions on the set of shifted primes has never been considered. We consider the case where the strongly additive functions on the set of primes take only two values 0 and 1 . To find weak convergence conditions, we use the method of factorial moments.

Throughout the paper we will keep the following notation. $p$ and $q$ with or without subscripts mean prime numbers; $c, c_{1}, c_{2}, \ldots$ are absolute positive constants. We denote by $\varepsilon(x)$ any quantity vanishing as $x \rightarrow \infty$. The notation $a \ll b$ is equivalent to the inequality $|a| \leq c b$ for some $c$. If a constant $c$, a constant implied by $\ll$, or a vanishing function $\varepsilon(x)$ depend on a parameter $a$, we write $c_{a}, \ll_{a}, \varepsilon_{a}(x)$. The notation $F_{x}(u) \Rightarrow F(u)$ means that the set of distribution functions $F_{x}(u)$ converges weakly to the distribution function $F(u)$ as $x \rightarrow \infty$. The Poisson distribution function with parameter $\lambda(\lambda>0)$ is denoted by $\Pi_{\lambda}(u)$ and the classical Euler function by $\phi$. The superscript ${ }^{f}$ in $\sum^{f}, \max ^{f}$ means that the summation or maximum is extended over primes $q$ for which $f_{x}(q)=1$. Other notation is generally accepted or is later discussed in the text.

\section{Main result and examples}

TheOREM. Let $f_{x}$ and $g_{x}, x \geq 2$, be two sets of strongly additive functions such that

$$
f_{x}(p), g_{x}(p) \in\{0,1\}
$$

for all primes $p$ and

$$
\lim _{x \rightarrow \infty}(\log x)\left(\sum_{x^{\alpha}<q \leq x}^{f} \frac{1}{q}+\sum_{x^{\alpha}<q \leq x}^{g} \frac{1}{q}\right)=0
$$

for all $\alpha \in(0,1)$. In order that

$$
\nu_{x}\left(p \leq x, f_{x}(p+1)+g_{x}(p+2)<u\right) \Rightarrow \Pi_{\lambda}(u)
$$


as $x \rightarrow \infty$ it is both necessary and sufficient that

$$
\begin{aligned}
& \lim _{x \rightarrow \infty}\left(\sum_{q \leq x}^{f} \frac{1}{q}+\sum_{q \leq x}^{g} \frac{1}{q}\right)=\lambda, \\
& \lim _{x \rightarrow \infty}\left(\max _{q \leq x} f \frac{1}{q}+\max _{q \leq x} g \frac{1}{q}\right)=0 .
\end{aligned}
$$

ExAMPLE 1. Let $\psi_{f}(x)$ and $\psi_{g}(x)$ be unboundedly increasing functions such that $\log \psi_{f}(x) / \log x \rightarrow 0$ and $\log \psi_{g}(x) / \log x \rightarrow 0$ as $x \rightarrow \infty$. Let

$$
f_{x}(p)= \begin{cases}1 & \text { if } \psi_{f}(x)<p \leq \psi_{f}^{\alpha}(x) \\ 0 & \text { otherwise }\end{cases}
$$

and

$$
g_{x}(p)= \begin{cases}1 & \text { if } \psi_{g}(x)<p \leq \psi_{g}^{\beta}(x) \\ 0 & \text { otherwise }\end{cases}
$$

with some $\alpha, \beta>1$. It follows from the Theorem that

$$
\lim _{x \rightarrow \infty} \nu_{x}\left(p \leq x, f_{x}(p+1)+g_{x}(p+2)=k\right)=\frac{(\log \alpha \beta)^{k}}{\alpha \beta k !}
$$

for every fixed $k=0,1,2, \ldots$ If $k=0$ we have

$\#\left\{\begin{array}{l|l}p \leq x & \begin{array}{l}p+1 \text { has no prime factors in the interval }\left(\psi_{f}(x), \psi_{f}^{\alpha}(x)\right] \\ p+2 \text { has no prime factors in }\left(\psi_{g}(x), \psi_{g}^{\beta}(x)\right]\end{array}\end{array}\right\}$

$$
\sim \frac{x}{\alpha \beta \log x}
$$

as $x \rightarrow \infty$.

EXAMPLE 2. Let

$$
f_{x}(p)=g_{x}(p)= \begin{cases}1 & \text { if } \log x<p \leq(\log x)^{2} \\ 0 & \text { otherwise }\end{cases}
$$

The Theorem implies that

$$
\lim _{x \rightarrow \infty} \nu_{x}\left(p \leq x, f_{x}(p+1)+g_{x}(p+2)=k\right)=\frac{(\log 4)^{k}}{4 k !}
$$

for every fixed $k=0,1,2, \ldots$ In case $k=0$, we get

$$
\#\left\{\begin{array}{l|l}
p \leq x & \begin{array}{l}
p+1 \text { and } p+2 \text { have no prime factors } \\
\text { in }\left(\log x,(\log x)^{2}\right]
\end{array}
\end{array}\right\} \sim \frac{x}{4 \log x}
$$

as $x \rightarrow \infty$. If $k=1$ we have

$$
\#\left\{\begin{array}{l|l}
p \leq x & \begin{array}{l}
\text { exactly one prime in }\left(\log x,(\log x)^{2}\right] \\
\text { divides } p+1 \text { or } p+2
\end{array}
\end{array}\right\} \sim \frac{x \log 4}{4 \log x}
$$

as $x \rightarrow \infty$. 


\section{Auxiliary lemmas}

Lemma 1 ([4]). For every real-valued additive function $h$, every real number $b$ and every integer $a$,

$$
\nu_{x}(p \leq x, h(p+a)=b) \ll\left(4+\sum_{\substack{q \leq x \\ h(q) \neq 0}} \frac{1}{q}\right)^{-1 / 2} .
$$

Lemma 2 ([5, Theorem 3.7]). The inequality

$$
\pi(x, d, v):=\sum_{\substack{p \leq x \\ p \equiv v \bmod d}} 1 \ll \frac{x}{\phi(d) \log \frac{x}{d}}
$$

holds uniformly for all $x \geq 2$ and integers $d, v$ with $(v, d)=1,1 \leq d<x$.

Lemma 3 ([1]). Let $K$ be a positive real number. Then there exists a real number $L$ such that uniformly for all $x \geq 2$,

$$
\sum_{d \leq x^{1 / 2}(\log x)^{-L}} \max _{(d, v)=1}\left|\pi(x, d, v)-\frac{\operatorname{li} x}{\phi(d)}\right| \ll \frac{x}{(\log x)^{K}} .
$$

Lemma 4. Let $f_{x}$ and $g_{x}, x \geq 2$, be two sets of strongly additive functions such that $f_{x}(p), g_{x}(p) \in\{0,1\}$ for all primes $p$ and

$$
\sum_{q \leq x}^{f} \frac{1}{q}+\sum_{q \leq x}^{g} \frac{1}{q} \leq c_{1}
$$

For positive integers $l$ let

$$
\begin{aligned}
\beta(l, x):= & \frac{1}{\pi(x)} \sum_{p \leq x}\left(f_{x}(p+1)+g_{x}(p+2)\right) \\
& \times\left(f_{x}(p+1)+g_{x}(p+2)-1\right) \ldots\left(f_{x}(p+1)+g_{x}(p+2)-l+1\right) .
\end{aligned}
$$

Then

$$
\beta(l, x) \ll_{l} c_{1}^{l}+c_{1}^{l-1}+\cdots+c_{1}+1 .
$$

Proof. We have

$$
\begin{aligned}
\beta(1, x)= & \frac{1}{\pi(x)} \sum_{p \leq x}\left(f_{x}(p+1)+g_{x}(p+2)\right)=\frac{1}{\pi(x)} \sum_{p \leq x}\left(\sum_{q \mid p+1}^{f} 1+\sum_{q \mid p+2}^{g} 1\right) \\
= & \frac{1}{\pi(x)} \sum_{q \leq \sqrt{x}}^{f} \pi(x, q,-1)+\frac{1}{\pi(x)} \sum_{p \leq x} \sum_{\substack{q>\sqrt{x} \\
q \mid p+1}}^{f} 1 \\
& +\frac{1}{\pi(x)} \sum_{q \leq \sqrt{x}}^{g} \pi(x, q,-2)+\frac{1}{\pi(x)} \sum_{\substack { p \leq x \\
\begin{subarray}{c}{q>\sqrt{x} \\
q \mid p+2{ p \leq x \\
\begin{subarray} { c } { q > \sqrt { x } \\
q | p + 2 } }\end{subarray}} \sum^{g} 1 .
\end{aligned}
$$


Since

$$
\sum_{\substack{q>\sqrt{x} \\ q \mid p+a}} 1 \leq 1
$$

for $p \leq x$ and for $a=1$ or 2 , Lemma 2 and the condition (5) imply that

$$
\beta(1, x) \ll c_{1}+1 \text {. }
$$

Assume now $l \in \mathbb{N}, l \geq 2$. It follows from the known combinatorial equalities that

$$
\begin{aligned}
& \beta(l, x)=\frac{1}{\pi(x)} \sum_{p \leq x} f_{x}(p+1)\left(f_{x}(p+1)-1\right) \ldots\left(f_{x}(p+1)-l+1\right) \\
& +\frac{1}{\pi(x)} \sum_{p \leq x} g_{x}(p+2)\left(g_{x}(p+2)-1\right) \ldots\left(g_{x}(p+2)-l+1\right) \\
& \quad+\sum_{k=1}^{l-1}\left(\begin{array}{l}
l \\
k
\end{array}\right) \frac{1}{\pi(x)} \sum_{p \leq x} f_{x}(p+1)\left(f_{x}(p+1)-1\right) \ldots\left(f_{x}(p+1)-k+1\right) \\
& \quad \times g_{x}(p+2)\left(g_{x}(p+2)-1\right) \ldots\left(g_{x}(p+2)-(l-k)+1\right) .
\end{aligned}
$$

The strong additivity of $f_{x}$ and $g_{x}$ implies that

$$
\begin{aligned}
f_{x}(p+1) & \ldots\left(f_{x}(p+1)-k+1\right) g_{x}(p+2) \ldots\left(g_{x}(p+2)-(l-k)+1\right) \\
& =\sum_{q_{1} \mid p+1}^{f} \sum_{\substack{q_{2} \mid p+1 \\
q_{2} \neq q_{1}}}^{f} \cdots \sum_{\substack{q_{k} \mid p+1 \\
q_{k} \neq q_{1}, \ldots, q_{k-1}}}^{f} \sum_{\substack{q_{k+1} \mid p+2 \\
q_{q_{k+2} \mid p+2} \\
q_{k+2} \neq q_{k+1}}}^{g} \ldots \sum_{\substack{q_{l} \mid p+2 \\
q_{l} \neq q_{k+1}, \ldots, q_{l-1}}}^{g} 1 \\
& =k !(l-k) ! \sum_{\substack{q_{1} \ldots q_{k} \mid p+1 \\
q_{1}<\cdots<q_{k}}}^{f} \sum_{\substack{q_{k+1} \ldots q_{l} \mid p+2 \\
q_{k+1}<\cdots<q_{l}}}^{g} 1,
\end{aligned}
$$

where the last double summation is extended over all collections of primes $q_{1}, \ldots, q_{k}, \ldots, q_{l}, q_{1}<\cdots<q_{k}, q_{k+1}<\cdots<q_{l}$ such that $f_{x}\left(q_{1}\right)=\cdots=$ $f_{x}\left(q_{k}\right)=g_{x}\left(q_{k+1}\right)=\cdots=g_{x}\left(q_{l}\right)=1$. Continuing the equality (8), we have

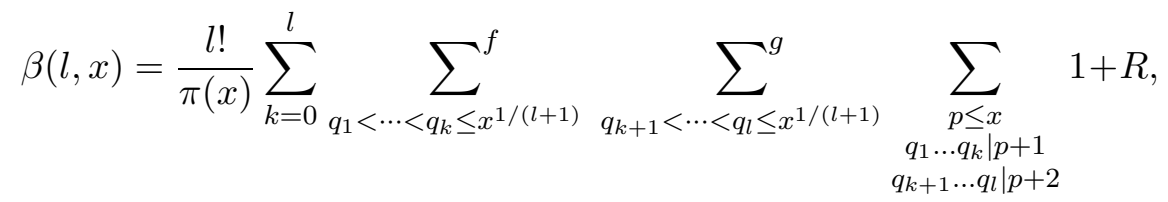

where

$$
R=O_{l}\left(\frac{1}{\pi(x)} \sum_{k=0}^{l} \sum_{p \leq x}\left(\sum_{\substack{q_{1} \ldots q_{k} \mid p+1 \\ q_{1}<\cdots<q_{k} \\ q_{k}>x^{1 /(l+1)}}}^{f} \sum_{\substack{q_{k+1} \ldots q_{l} \mid p+2 \\ q_{k+1}<\cdots<q_{l}}}^{g} 1+\sum_{\substack{q_{1} \ldots q_{k} \mid p+1 \\ q_{1}<\cdots<q_{k}}}^{f} \sum_{\substack{q_{k+1} \ldots q_{l} \mid p+2 \\ q_{k+1}<\cdots<q_{l} \\ q_{l}>x^{1 /(l+1)}}}^{g} 1\right)\right) .
$$


Since

$$
\sum_{\substack{q>x^{1 /(l+1)} \\ q \mid p+a}} 1 \ll_{l} 1
$$

for $p \leq x$ and for $a=1$ or 2 , we have

$$
R \ll_{l} \beta(l-1, x) .
$$

On the other hand, for any positive integers $k_{1}, k_{2}$,

$$
\sum_{\substack{p \leq x \\ k_{1}\left|p+1 \\ k_{2}\right| p+2}} 1=\pi\left(x, k_{1} k_{2}, v\right)
$$

where $v$ is the only integer for which

$$
v \equiv-2 \bmod k_{1}, \quad v \equiv-1 \bmod k_{2},
$$

and $1 \leq v \leq k_{1} k_{2}-1$. Therefore, according to the estimate of Lemma 2, we have

$$
\begin{aligned}
\beta(l, x) & \ll_{l} \beta(l-1, x)+\sum_{k=0}^{l} \sum_{q_{1}<\cdots<q_{k} \leq x^{1 /(l+1)}}^{f} \sum_{q_{k+1}<\cdots<q_{l} \leq x^{1 /(l+1)}}^{g} \frac{1}{\phi\left(q_{1} \ldots q_{l}\right)} \\
& \ll_{l} \beta(l-1, x)+\left(\sum_{q \leq x^{1 /(l+1)}}^{f} \frac{1}{q}+\sum_{q \leq x^{1 /(l+1)}}^{g} \frac{1}{q}\right)^{l} .
\end{aligned}
$$

Using the condition (5), we obtain

$$
\beta(l, x) \ll_{l} c_{1}^{l}+\beta(l-1, x) .
$$

The estimates (7) and (11) now imply the inequality (6).

4. Proof of Theorem. Necessity. The condition $(\mathrm{H})$ implies that there exists a vanishing function $\alpha(x)$ such that

$$
\lim _{x \rightarrow \infty} x^{\alpha(x)}=\infty
$$

and

$$
\lim _{x \rightarrow \infty}(\log x)\left(\sum_{x^{\alpha(x)}<q \leq x}^{f} \frac{1}{q}+\sum_{x^{\alpha(x)}<q \leq x}^{g} \frac{1}{q}\right)=0 .
$$

Let $\widehat{f}_{x}$ and $\widehat{g}_{x}$ be two new sets of strongly additive functions defined by the equalities:

$$
\widehat{f}_{x}(q)=\left\{\begin{array}{ll}
f_{x}(q) & \text { if } q \leq x^{\alpha(x)}, \\
0 & \text { if } q>x^{\alpha(x)},
\end{array} \quad \widehat{g}_{x}(q)= \begin{cases}g_{x}(q) & \text { if } q \leq x^{\alpha(x)} \\
0 & \text { if } q>x^{\alpha(x)}\end{cases}\right.
$$


For every positive $\varepsilon$,

$$
\begin{aligned}
\nu_{x}(p \leq x, & \left.\left|f_{x}(p+1)+g_{x}(p+2)-\widehat{f}_{x}(p+1)-\widehat{g}_{x}(p+2)\right|>\varepsilon\right) \\
\leq & \nu_{x}\left(p \leq x,\left|f_{x}(p+1)-\widehat{f}_{x}(p+1)\right|>\varepsilon / 2\right) \\
& +\nu_{x}\left(p \leq x,\left|g_{x}(p+2)-\widehat{g}_{x}(p+2)\right|>\varepsilon / 2\right) \\
\leq & \nu_{x}\left(p \leq x, \exists q \mid p+1: f_{x}(q) \neq \widehat{f}_{x}(q)\right) \\
& +\nu_{x}\left(p \leq x, \exists q \mid p+2: g_{x}(q) \neq \widehat{g}_{x}(q)\right) .
\end{aligned}
$$

The first summand of the right hand side above does not exceed

$$
\begin{aligned}
\frac{1}{\pi(x)} \sum_{x^{\alpha(x)}<q \leq x+1} \sum_{\substack{p \leq x \\
q \mid p+1}} 1 & \leq \frac{1}{\pi(x)} \sum_{x^{\alpha(x)}<q \leq x+1}^{f} \sum_{\substack{n \leq x \\
q \mid n+1}} 1 \\
& \ll(\log x)\left(\sum_{x^{\alpha(x)<q \leq x}}^{f} \frac{1}{q}+\frac{1}{x}\right) .
\end{aligned}
$$

An analogous estimate holds for the second summand of the right hand side of (13). Thus

$$
\begin{aligned}
\nu_{x}\left(p \leq x, \mid f_{x}(p+1)+\right. & \left.g_{x}(p+2)-\widehat{f}_{x}(p+1)-\widehat{g}_{x}(p+2) \mid>\varepsilon\right) \\
& \ll(\log x)\left(\sum_{x^{\alpha(x)}<q \leq x}^{f} \frac{1}{q}+\sum_{x^{\alpha(x)}<q \leq x}^{g} \frac{1}{q}+\frac{1}{x}\right) .
\end{aligned}
$$

Hence (12) and the weak convergence condition (2) imply that

$$
\nu_{x}\left(p \leq x, \widehat{f}_{x}(p+1)+\widehat{g}_{x}(p+2)<u\right) \underset{x \rightarrow \infty}{\Rightarrow} \Pi_{\lambda}(u)
$$

It follows that

$$
\begin{aligned}
\liminf _{x \rightarrow \infty} \nu_{x}\left(p \leq x, \widehat{f}_{x}\right. & (p+1)=0) \\
& \geq \liminf _{x \rightarrow \infty} \nu_{x}\left(p \leq x, \widehat{f}_{x}(p+1)+\widehat{g}_{x}(p+2)=0\right)=e^{-\lambda} .
\end{aligned}
$$

Therefore, according to Lemma 1 ,

$$
\sum_{q \leq x^{\alpha(x)}}^{f} \frac{1}{q} \leq c_{2, \lambda} .
$$

Similarly

$$
\sum_{q \leq x^{\alpha(x)}}^{g} \frac{1}{q} \leq c_{3, \lambda} .
$$

Hence, applying Lemma 4, we get

$$
\widehat{\beta}(l, x) \ll_{l, \lambda} 1,
$$


where

$\widehat{\beta}(l, x)=\frac{1}{\pi(x)} \sum_{p \leq x}\left(\widehat{f}_{x}(p+1)+\widehat{g}_{x}(p+2)\right) \ldots\left(\widehat{f}_{x}(p+1)+\widehat{g}_{x}(p+2)-l+1\right)$.

From the weak convergence (15) we infer that, for every $k \in\{0\} \cup \mathbb{N}$,

$$
\frac{1}{\pi(x)} \sum_{\substack{p \leq x \\ \widehat{f}_{x}(p+1)+\widehat{g}_{x}(p+2)=k}} 1=\frac{\lambda^{k}}{k !} e^{-\lambda}+\varepsilon_{k}(x) .
$$

Let us fix $l \in \mathbb{N}$ and choose $K>l+2$. Using the estimate (16) we obtain

$$
\begin{aligned}
\widehat{\beta}(l, x)= & \sum_{k=l}^{K} k(k-1) \ldots(k-l+1) \frac{1}{\pi(x)} \sum_{\substack{p \leq x \\
\widehat{f}_{x}(p+1)+\widehat{g}_{x}(p+2)=k}} 1 \\
& +\frac{1}{\pi(x)} \sum_{\substack{p \leq x \\
\widehat{f}_{x}(p+1)+\widehat{g}_{x}(p+2)>K}}\left(\widehat{f}_{x}(p+1)+\widehat{g}_{x}(p+2)\right) \ldots \\
& \ldots\left(\widehat{f}_{x}(p+1)+\widehat{g}_{x}(p+2)-l+1\right) \frac{\widehat{f}_{x}(p+1)+\widehat{g}_{x}(p+2)-l}{\widehat{f}_{x}(p+1)+\widehat{g}_{x}(p+2)-l} \\
= & \sum_{k=l}^{K} k_{(k-1) \ldots(k-l+1)} \frac{\lambda^{k}}{k !} e^{-\lambda+\varepsilon_{K, l}(x)+O\left(\frac{\beta}{k}(l+1, x)\right.} \\
= & \lambda^{l}+O_{\lambda}\left(\frac{\lambda^{K+1}}{(K+1-l) !}\right)+O_{l, \lambda}\left(\frac{1}{K-l}\right)+\varepsilon_{K, l}(x) .
\end{aligned}
$$

From this equality it follows that

$$
\lim _{x \rightarrow \infty} \widehat{\beta}(l, x)=\lambda^{l}
$$

for every fixed positive integer $l$. If $l=1$, from (17) we have

$$
\lim _{x \rightarrow \infty} \widehat{\beta}(1, x)=\lambda \text {. }
$$

On the other hand,

$$
\begin{aligned}
\widehat{\beta}(1, x)= & \frac{\operatorname{li} x}{\pi(x)} \sum_{q \leq x^{\alpha(x)}}^{f} \frac{1}{\phi(q)}+\frac{1}{\pi(x)} \sum_{q \leq x^{\alpha(x)}}^{f}\left(\pi(x, q,-1)-\frac{\operatorname{li} x}{\phi(q)}\right) \\
& +\frac{\operatorname{li} x}{\pi(x)} \sum_{q \leq x^{\alpha(x)}}^{g} \frac{1}{\phi(q)}+\frac{1}{\pi(x)} \sum_{q \leq x^{\alpha(x)}}^{g}\left(\pi(x, q,-2)-\frac{\operatorname{li} x}{\phi(q)}\right) .
\end{aligned}
$$

In view of Lemma 3, it follows from the last two equalities that

$$
\lim _{x \rightarrow \infty}\left(\sum_{q \leq x^{\alpha(x)}}^{f} \frac{1}{\phi(q)}+\sum_{q \leq x^{\alpha(x)}}^{g} \frac{1}{\phi(q)}\right)=\lambda \text {. }
$$


If $l=2$, from (17) we have

$$
\lim _{x \rightarrow \infty} \widehat{\beta}(2, x)=\lambda^{2} .
$$

The value of $\widehat{\beta}(2, x)$ can also be written in the following way:

$$
\begin{aligned}
& \widehat{\beta}(2, x)=\frac{1}{\pi(x)} \sum_{p \leq x} \widehat{f}_{x}(p+1)\left(\widehat{f}_{x}(p+1)-1\right) \\
& +\frac{2}{\pi(x)} \sum_{p \leq x} \widehat{f}_{x}(p+1) \widehat{g}_{x}(p+2)+\frac{1}{\pi(x)} \sum_{p \leq x} \widehat{g}_{x}(p+2)\left(\widehat{g}_{x}(p+2)-1\right) \\
& =\frac{2}{\pi(x)} \sum_{q_{1}<q_{2} \leq x^{\alpha(x)}}^{f} \sum_{\substack{p \leq x \\
q_{1} q_{2} \mid p+1}} 1+\frac{2}{\pi(x)} \sum_{q_{1} \leq x^{\alpha(x)}}^{f} \sum_{q_{2} \leq x^{\alpha(x)}}^{g} \sum_{\substack{p \leq x \\
q_{1}\left|p+1 \\
q_{2}\right| p+2}} 1 \\
& +\frac{2}{\pi(x)} \sum_{q_{1}<q_{2} \leq x^{\alpha(x)}}^{g} \sum_{\substack{p \leq x \\
q_{1} q_{2} \mid p+2}} 1=: S_{1}+S_{2}+S_{3} .
\end{aligned}
$$

According to Lemma 3,

$$
\begin{aligned}
S_{1} & =\frac{2 \operatorname{li} x}{\pi(x)} \sum_{q_{1}<q_{2} \leq x^{\alpha(x)}}^{f} \frac{1}{\phi\left(q_{1} q_{2}\right)}+\frac{2}{\pi(x)} \sum_{q_{1}<q_{2} \leq x^{\alpha(x)}}^{f}\left(\pi\left(x, q_{1} q_{2},-1\right)-\frac{\operatorname{li} x}{\phi\left(q_{1} q_{2}\right)}\right) \\
& =\left(2+O\left(\frac{1}{\log x}\right)\right) \sum_{q_{1}<q_{2} \leq x^{\alpha(x)}}^{f} \frac{1}{\phi\left(q_{1} q_{2}\right)}+O\left(\frac{1}{\log x}\right) .
\end{aligned}
$$

Similarly

$$
S_{3}=\left(2+O\left(\frac{1}{\log x}\right)\right) \sum_{q_{1}<q_{2} \leq x^{\alpha(x)}}^{g} \frac{1}{\phi\left(q_{1} q_{2}\right)}+O\left(\frac{1}{\log x}\right) .
$$

Finally, using (10) and Lemma 3 once again, we see that $S_{2}$ can be written as

$$
\begin{aligned}
S_{2} & =\frac{2}{\pi(x)} \sum_{q_{1} \leq x^{\alpha(x)}}^{f} \sum_{\substack{q_{2} \leq x^{\alpha(x)} \\
q_{2} \neq q_{1}}}^{g} \pi\left(x, q_{1} q_{2}, v\right) \\
& =2\left(1+O\left(\frac{1}{\log x}\right)\right) \sum_{\substack{q_{1} \leq x^{\alpha(x)} \\
q_{2} \leq x^{\alpha(x)} \\
q_{2} \neq q_{1}}}^{f} \frac{1}{\phi\left(q_{1} q_{2}\right)}+O\left(\frac{1}{\log x}\right) .
\end{aligned}
$$


From (19), (20) and the expressions for $S_{1}, S_{2}$, and $S_{3}$, we have

$$
\begin{aligned}
& \lim _{x \rightarrow \infty}\left(2 \sum_{q_{1}<q_{2} \leq x^{\alpha(x)}}^{f} \frac{1}{\phi\left(q_{1} q_{2}\right)}+2 \sum_{q_{1} \leq x^{\alpha(x)}}^{f} \sum_{q_{2} \leq x^{\alpha(x)}}^{g} \frac{1}{\phi\left(q_{1} q_{2}\right)}+2 \sum_{q_{1}<q_{2} \leq x^{\alpha(x)}}^{g} \frac{1}{\phi\left(q_{1} q_{2}\right)}\right) \\
& q_{2} \neq q_{1} \\
& =\lambda^{2} \text {. }
\end{aligned}
$$

Hence

$$
\begin{aligned}
\lambda^{2}= & \left(\sum_{q \leq x^{\alpha(x)}}^{f} \frac{1}{\phi(q)}+\sum_{q \leq x^{\alpha(x)}}^{g} \frac{1}{\phi(q)}\right)^{2} \\
& -\sum_{q \leq x^{\alpha(x)}}^{f} \frac{1}{\phi^{2}(q)}-2 \sum_{\substack{q \leq x^{\alpha(x)} \\
f_{x}(q)=g_{x}(q)=1}} \frac{1}{\phi^{2}(q)}-\sum_{q \leq x^{\alpha(x)}}^{g} \frac{1}{\phi^{2}(q)}+\varepsilon(x) .
\end{aligned}
$$

The last expression and the equality (18) imply that

$$
\lim _{x \rightarrow \infty}\left(\max _{q \leq x^{\alpha(x)}} f \frac{1}{\phi(q)}+\max _{q \leq x^{\alpha(x)}} \frac{1}{\phi(q)}\right)=0 .
$$

The relations (3) and (4) now follow from (18), (21), and (12). The necessity is proved.

5. Proof of Theorem. Sufficiency. Assume that the conditions (3), (4) and the additional condition $(\mathrm{H})$ are satisfied. Let $\alpha(x)$ be the same vanishing function and $\widehat{f}_{x}, \widehat{g}_{x}$ be the same strongly additive functions as in the previous section. According to (14), it is enough to prove (15).

Put

$$
\psi_{x}(t)=\frac{1}{\pi(x)} \sum_{p \leq x} e^{i t\left(\widehat{f}_{x}(p+1)+\widehat{g}_{x}(p+2)\right)}
$$

for $x \geq 2$ and $t \in \mathbb{R}$. Because for all $r \in\{0\} \cup \mathbb{N}$ and $L \in \mathbb{N}$,

$$
\left|e^{i t r}-1-\sum_{l=1}^{L}\left(\begin{array}{l}
r \\
l
\end{array}\right)\left(e^{i t}-1\right)^{l}\right| \leq\left(\begin{array}{c}
r \\
L+1
\end{array}\right)\left|e^{i t}-1\right|^{L+1},
$$

we have

$$
\psi_{x}(t)=1+\sum_{l=1}^{L} \frac{\widehat{\beta}(l, x)}{l !}\left(e^{i t}-1\right)^{l}+O\left(\frac{\widehat{\beta}(L+1, x)}{(L+1) !}\left|e^{i t}-1\right|^{L+1}\right)
$$

for all positive integers $L$.

If $l$ is fixed and $x$ is sufficiently large, arguing as in (8) and (9), we obtain

$$
\widehat{\beta}(l, x)=\frac{l !}{\pi(x)} \sum_{k=0}^{l} \sum_{q_{1}<\cdots<q_{k} \leq x^{\alpha(x)}}^{f} \sum_{q_{k+1}<\cdots<q_{l} \leq x^{\alpha(x)}}^{g} \sum_{\substack{p \leq x \\ q_{1} \ldots q_{k}\left|p+1 \\ q_{k+1} \ldots q_{l}\right| p+2}} 1 .
$$


The use of (10) enables writing $\widehat{\beta}(l, x)$ as a sum of two sums:

$$
\begin{gathered}
\widehat{\beta}(l, x)=\frac{l ! \operatorname{li} x}{\pi(x)} \sum_{k=0}^{l} \sum_{q_{1}<\cdots<q_{k} \leq x^{\alpha(x)}}^{f} \sum_{\substack{q_{k+1}<\cdots<q_{l} \leq x^{\alpha(x)} \\
q_{k+1}, \ldots, q_{l} \neq q_{1}, \ldots, q_{k}}}^{g} \frac{1}{\phi\left(q_{1} \ldots q_{l}\right)} \\
+\frac{l !}{\pi(x)} \sum_{k=0}^{l} \sum_{\substack{q_{1}<\cdots<q_{k} \leq x^{\alpha(x)} \\
q_{k+1}<\cdots<q_{l} \leq x^{\alpha(x)} \\
q_{k+1}, \ldots, q_{l} \neq q_{1}, \ldots, q_{k}}}^{g}\left(\pi\left(x, q_{1} \ldots q_{l}, v\right)-\frac{\operatorname{li} x}{\phi\left(q_{1} \ldots q_{l}\right)}\right) \\
=: S_{4}+S_{5} .
\end{gathered}
$$

We have

$$
\begin{aligned}
S_{4}= & \left(1+O\left(\frac{1}{\log x}\right)\right) \sum_{k=0}^{l}\left(\begin{array}{l}
l \\
k
\end{array}\right)\left(\sum_{q \leq x^{\alpha(x)}}^{f} \frac{1}{\phi(q)}\right)^{k}\left(\sum_{q \leq x^{\alpha(x)}}^{g} \frac{1}{\phi(q)}\right)^{l-k} \\
& +\max \left\{\max _{q \leq x^{\alpha(x)}}^{f} \frac{1}{\phi(q)}, \max _{q \leq x^{\alpha(x)}}^{g} \frac{1}{\phi(q)}\right\} \\
& \times O_{l}\left(\left(\max \left\{1, \sum_{q \leq x^{\alpha(x)}}^{f} \frac{1}{\phi(q)}, \sum_{q \leq x^{\alpha(x)}}^{g} \frac{1}{\phi(q)}\right\}\right)^{l-1}\right) .
\end{aligned}
$$

By Lemma 3,

$$
S_{5}=O_{l}\left(\frac{1}{\log x}\right) .
$$

The conditions (3), (4), and (H) immediately yield (18) and (21). These equalities together with (23) and (24) now imply that

$$
\lim _{x \rightarrow \infty} \widehat{\beta}(l, x)=\lambda^{l}
$$

for every fixed positive integer $l$. Hence applying (22), we have

$$
\begin{aligned}
\limsup _{x \rightarrow \infty}\left|\psi_{x}(t)-e^{\lambda\left(e^{i t}-1\right)}\right| & =\limsup _{x \rightarrow \infty}\left|\sum_{l=L+1}^{\infty} \frac{\lambda^{l}}{l !}\left(e^{i t}-1\right)^{l}\right|+O\left(\frac{(2 \lambda)^{L+1}}{(L+1) !}\right) \\
& =O_{\lambda}\left(\frac{(2 \lambda)^{L+1}}{(L+1) !}\right)
\end{aligned}
$$

for all real $t$. Letting $L \rightarrow \infty$, we obtain

$$
\lim _{x \rightarrow \infty} \psi_{x}(t)=e^{\lambda\left(e^{i t}-1\right)}
$$

and this implies the weak convergence (15). The Theorem is proved.

\section{References}

[1] E. Bombieri, On large sieve, Mathematika 12 (1965), 201-225.

[2] P. D. T. A. Elliott, Probabilistic Number Theory. I, Springer, Berlin, 1979. 
[3] P. D. T. A. Elliott, Probabilistic Number Theory. II, Springer, Berlin, 1980.

[4] - The concentration function of additive functions on shifted primes, Acta Math. 173 (1994), 1-35.

[5] H. Halberstam and H.-E. Richert, Sieve Methods, Academic Press, London, 1974.

[6] I. Kátai, On the distribution of arithmetical functions on the set of primes plus one, Compos. Math. 19 (1968), 278-289.

[7] J. Kubilius, Probabilistic Methods in the Theory of Numbers, Amer. Math. Soc. Transl. Math. Monogr. 11, Providence, 1964.

[8] J. Šiaulys and G. Stepanauskas, The Poisson distribution for a sum of additive functions, Acta Appl. Math. 97 (2007), 269-279.

[9] - - - The Poisson law for additive functions on shifted primes, in: Analytic and Probabilistic Methods in Number Theory, in Honour of J. Kubilius (Palanga, 2006), E. Manstavičius and A. Laurinčikas (eds.), TEV, Vilnius, 2007, in print.

Department of Probability Theory

and Number Theory

Vilnius University

Naugarduko St. 24

LT-03225 Vilnius, Lithuania

E-mail: jonas.siaulys@mif.vu.lt
Department of Mathematical Computer Science Vilnius University Naugarduko St. 24

LT-03225 Vilnius, Lithuania E-mail: gediminas.stepanauskas@maf.vu.lt 